PROCEEDINGS OF THE

AMERICAN MATHEMATICAL SOCIETY

Volume 139, Number 4, April 2011, Pages 1383-1386

S 0002-9939(2010)10544-0

Article electronically published on August 23, 2010

\title{
ON SPACES OF COMPACT OPERATORS ON $C(K, X)$ SPACES
}

\author{
ELÓI MEDINA GALEGO
}

(Communicated by Nigel J. Kalton)

\begin{abstract}
This paper concerns the spaces of compact operators $\mathcal{K}(E, F)$, where $E$ and $F$ are Banach spaces $C([1, \xi], X)$ of all continuous $X$-valued functions defined on the interval of ordinals $[1, \xi]$ and equipped with the supremun norm. We provide sufficient conditions on $X, Y, \alpha, \beta, \xi$ and $\eta$, with $\omega \leq \alpha \leq \beta<\omega_{1}$ for the following equivalence:

(a) $\mathcal{K}(C([1, \xi], X), C([1, \alpha], Y))$ is isomorphic to $\mathcal{K}(C([1, \eta], X), C([1, \beta], Y))$, (b) $\beta<\alpha^{\omega}$.

In this way, we unify and extend results due to Bessaga and Pełczyński (1960) and C. Samuel (2009). Our result covers the case of the classical spaces $X=l_{p}$ and $Y=l_{q}$, with $1<p, q<\infty$.
\end{abstract}

\section{INTRODUCTION}

We use standard Banach space theory terminology and notions as can be found in 12 and 13. Let $X$ be a Banach space and $K$ a compact Hausdorff space. By $C(K, X)$ we denote the Banach space of all continuous $X$-valued functions defined on $K$ and equipped with the supremum norm. This space will be denoted by $C(K)$ in the case $X=\mathbb{R}$. Given Banach spaces $X$ and $Y, \mathcal{K}(X, Y)$ denotes the Banach space of compact operators from $X$ to $Y$. We write $X \sim Y$ when the Banach spaces $X$ and $Y$ are isomorphic and $X \hookrightarrow Y$ when $Y$ has a subspace isomorphic to $X$. Let $\alpha$ be an ordinal number. By $[1, \alpha]$ we denote the interval of ordinals $\{\xi: 1 \leq \xi \leq \alpha\}$ endowed with the order topology.

The present paper is devoted to the isomorphic classifications of spaces of compact operators from $C([1, \xi], X)$ to $C([1, \eta], Y)$ spaces, with $\eta<\omega_{1}$. A fundamental result along these lines is the classical Bessaga-Pełczyński 1960 isomorphic classification of $C([1, \alpha])$ spaces, with $\alpha<\omega_{1}$ [1, Theorem 4.1], that is:

Theorem 1.1. Suppose that $\omega \leq \alpha \leq \beta<\omega_{1}$. Then we have

$$
C([1, \alpha]) \sim C([1, \beta]) \Longleftrightarrow \beta<\alpha^{\omega} .
$$

Very recently C. Samuel classified the $\mathcal{K}(C([1, \alpha]), C([1, \alpha]))$ spaces, with $\alpha<\omega_{1}$ [15. Theorem 3.3] by proving:

Theorem 1.2. Suppose that $\omega \leq \alpha \leq \beta<\omega_{1}$. Then we have

$$
\mathcal{K}(C([1, \alpha]), C([1, \alpha])) \sim \mathcal{K}(C([1, \beta]), C([1, \beta])) \Longleftrightarrow \beta<\alpha^{\omega} .
$$

Received by the editors November 8, 2009 and, in revised form, April 9, 2010 and April 16, 2010.

2010 Mathematics Subject Classification. Primary 46B03; Secondary 46B25.

Key words and phrases. Isomorphic classifications of spaces of compact operators.

(C)2010 American Mathematical Society 
The main aim of this short work is to unify and extend these results as follows.

Theorem 1.3. Let $\alpha, \beta, \xi$ and $\eta$ be ordinals with $\omega \leq \alpha \leq \beta<\omega_{1}$, $\xi \eta<\omega$ or else $\xi$ and $\eta$ of the same cardinality, $X$ a Banach space such that $X^{*}$ is weakly sequentially complete and has the approximation property and $Y$ a Banach space which has an unconditional basis and contains no subspace isomorphic to $l_{1}$. Then

$$
\mathcal{K}(C([1, \xi], X), C([1, \alpha], Y)) \sim \mathcal{K}(C([1, \eta], X), C([1, \beta], Y)) \Longleftrightarrow \beta<\alpha^{\omega} .
$$

Observe that Theorem 1.1 is the case $\xi=\eta=1$ and $X=Y=\mathbb{R}$ of Theorem 1.3. Moreover, Theorem 1.2 is the case $\alpha=\xi, \beta=\eta$ and $X=Y=\mathbb{R}$ of Theorem 1.3. Notice also that as an immediate consequence of Theorem 1.3 and Theorem 1.1 we get the following cancellation law:

Corollary 1.4. Let $\alpha, \beta, \xi$ and $\eta$ be ordinals with $\omega \leq \alpha \leq \beta<\omega_{1}, \xi \eta<\omega$ or else $\xi$ and $\eta$ of the same cardinality. Then for every $1<p, q<\infty$ we have

$\mathcal{K}\left(C\left([1, \xi], l_{p}\right), C\left([1, \alpha], l_{q}\right)\right) \sim \mathcal{K}\left(C\left([1, \eta], l_{p}\right), C\left([1, \beta], l_{q}\right)\right) \Longleftrightarrow C([1, \alpha]) \sim C([1, \beta])$.

This corollary gives a partial answer to [8, Problem 4.2.3]. We stress that the statement of Corollary 1.4 is also true for $1<p<\infty$ and $q=1$; see [8, Remark 4.1.3] for $\xi<\omega$ and [9, Remark 1.7] for $\xi \geq \omega$. Nevertheless the isomorphic classification of the $\mathcal{K}\left(l_{1}, l_{q}^{\alpha}\right)$ spaces, with $\omega \leq \alpha<\omega_{1}$ and $1 \leq q<\infty$, remains an open question; see [8, Problem 4.2.2]. In order to prove Theorem 1.3 we need some preliminary results.

\section{Preliminary Results}

From now on following [1] the $C([1, \alpha], X)$ spaces will be denoted by $X^{\alpha}$. One of the key steps in proving Theorem 1.3 is the following proposition. We will denote by $X \hat{\otimes} Y$ the injective tensor product of the Banach spaces $X$ and $Y$.

Proposition 2.1. Let $X$ be a weakly sequentially complete Banach space and $Y$ a Banach space which has an unconditional basis and contains no subspace isomorphic to $l_{1}$. Then for every set $\Gamma$ we have

$$
\mathbb{R}^{\omega^{\omega}} \hookrightarrow l_{1}(\Gamma, X) \hat{\hat{\otimes}} Y^{\omega}
$$

Proof. Suppose for contradiction that $l_{1}(\Gamma, X) \hat{\hat{\otimes}} Y^{\omega}$ has a subspace isomorphic to $\mathbb{R}^{\omega^{\omega}}$. Since $\mathbb{R}^{\omega^{\omega}}$ is separable, it is easily seen that

$$
\mathbb{R}^{\omega^{\omega}} \hookrightarrow l_{1}(\mathbb{N}, X) \hat{\hat{\otimes}} Y^{\omega}
$$

Assume that $Y$ has an unconditional basis and contains no subspace isomorphic to $l_{1}$. Then $Y^{\omega}$ does not contain a subspace isomorphic to $l_{1}$ (see for instance [7. Theorem 2.3]) and has an unconditional basis. So by a well known result of James [12, Theorem 1.c.9] $Y^{\omega}$ has a shrinking unconditional basis. Moreover, by [5. Corollary 1] $l_{1}(\mathbb{N}, X)$ is weakly sequentially complete. Therefore according to

[5. Theorem 3] $l_{1}(\mathbb{N}, X) \hat{\hat{\otimes}} Y^{\omega}$ has the property $(\mathrm{u})$ introduced by Pełczyński in [14, Definition 1]. Since this property is inherited by closed subspaces ([14, page 252] or [13. Proposition 1.c.3]), we would conclude by (1) that $\mathbb{R}^{\omega^{\omega}}$ has the property (u), which is absurd by an unpublished result of Pełczyński; see [10, pages 210-211] and [11, Proposition 5.3].

The next proposition is inspired by [15, Theorem 3.2]. 
Proposition 2.2. Let $\omega^{\omega} \leq \xi \leq \eta<\omega_{1}$ be two ordinals and $X$ a Banach space such that $X^{\omega}$ contains no subspace isomorphic to $\mathbb{R}^{\omega^{\omega}}$. If $\mathbb{R}^{\eta} \hookrightarrow X^{\xi}$, then $\mathbb{R}^{\eta} \hookrightarrow \mathbb{R}^{\xi}$.

Proof. We introduce two sets of ordinals:

$$
\begin{aligned}
& I_{1}=\left\{\theta: \omega^{\omega} \leq \theta<\omega_{1}, \quad \mathbb{R}^{\theta} \nrightarrow \mathbb{R}^{\gamma}, \forall \gamma<\theta\right\}, \\
& I_{2}=\left\{\theta: \omega^{\omega} \leq \theta<\omega_{1}, \quad \mathbb{R}^{\theta} \nrightarrow X^{\gamma}, \forall \gamma<\theta\right\} .
\end{aligned}
$$

First of all we will prove that $I_{1}=I_{2}$. Clearly $I_{2} \subset I_{1}$. Observe that by Theorem 1.1 and our hypothesis, we deduce that $\omega^{\omega} \in I_{2}$. Now, assume that $I_{2}$ is a proper subset of $I_{1}$. Let $\alpha_{1}$ be the least element of $I_{1} \backslash I_{2}$. We have $\omega^{\omega}<\alpha_{1}$. Since $\alpha_{1} \notin I_{2}$, there exists an ordinal $\gamma_{1}<\alpha_{1}$ such that $\mathbb{R}^{\alpha_{1}} \hookrightarrow X^{\gamma_{1}}$.

Let $\alpha_{2}=\min \left\{\gamma, \omega^{\omega} \leq \gamma<\alpha_{1}: \mathbb{R}^{\alpha_{1}} \hookrightarrow X^{\gamma}\right\}$. We have $\alpha_{2} \leq \gamma_{1}$. Now, we will show that $\alpha_{2} \in I_{1}$. If this is not the case, there exists an ordinal $\gamma_{2}<\alpha_{2}$ such that $\mathbb{R}^{\alpha_{2}} \hookrightarrow \mathbb{R}^{\gamma_{2}}$. Therefore $X^{\alpha_{2}} \hookrightarrow X^{\gamma_{2}}$. Consequently, $\mathbb{R}^{\alpha_{1}} \hookrightarrow X^{\gamma_{2}}$. This is a contradiction to the definition of $\alpha_{2}$.

So $\alpha_{2} \in I_{1}$ and since $\alpha_{2}<\alpha_{1}$, it follows from the definition of $\alpha_{1}$ that $\alpha_{2} \in I_{2}$. That is, $\mathbb{R}^{\alpha_{2}} \hookrightarrow X^{\gamma}, \forall \gamma<\alpha_{2}$. Thus by [6, Lemma 3.3], we conclude that $\mathbb{R}^{\alpha_{2}^{\omega}} \hookrightarrow$ $X^{\alpha_{2}}$.

On the other hand, note that if $\alpha_{1}<\alpha_{2}{ }^{\omega}$, then by Theorem 1.1, $\mathbb{R}^{\alpha_{1}} \sim \mathbb{R}^{\alpha_{2}}$, which is absurd by the definition of $\alpha_{1}$. Consequently $\alpha_{2}{ }^{\omega} \leq \alpha_{1}$ and $\mathbb{R}^{\alpha_{2}^{\omega}} \hookrightarrow \mathbb{R}^{\alpha_{1}}$. Furthermore, by the definition of $\alpha_{2}, \mathbb{R}^{\alpha_{1}} \hookrightarrow X^{\alpha_{2}}$. Therefore $\mathbb{R}^{\alpha_{2}^{\omega}} \hookrightarrow X^{\alpha_{2}}$, in contradiction to what we have proved above. Hence $I_{1}=I_{2}$.

Next, to complete the proof of the proposition, suppose that $\mathbb{R}^{\eta} \hookrightarrow \mathbb{R}^{\xi}$ and let $\xi_{1}=\min \left\{\theta: \mathbb{R}^{\eta} \hookrightarrow \mathbb{R}^{\theta}\right\}$. Hence $\xi<\xi_{1} \leq \eta$ and $\mathbb{R}^{\xi_{1}} \hookrightarrow \mathbb{R}^{\gamma}, \forall \gamma<\xi_{1}$. In particular, $\xi_{1} \in I_{1}=I_{2}$, which is absurd, because $\mathbb{R}^{\xi_{1}} \hookrightarrow \mathbb{R}^{\eta} \hookrightarrow X^{\xi}$.

\section{Proof of Theorem 1.3}

Initially notice that if $\gamma$ and $\theta$ are ordinals with $\gamma<\omega$ and $\omega \leq \theta<\omega_{1}$, then by Theorem 1.1, $\mathbb{R}^{\theta \gamma} \sim \mathbb{R}^{\theta}$. Therefore by [3, Proposition 5.3] we deduce that

$$
\mathcal{K}\left(X^{\gamma}, Y^{\theta}\right) \sim\left(X^{*}\right)^{\gamma} \hat{\hat{\otimes}} Y^{\theta} \sim X^{*} \hat{\hat{\otimes}} Y \hat{\hat{\otimes}} \mathbb{R}^{\gamma} \hat{\hat{\otimes}} \mathbb{R}^{\theta} \sim X^{*} \hat{\hat{\otimes}} Y \hat{\hat{\otimes}} \mathbb{R}^{\theta \gamma} \sim X^{*} \hat{\hat{\otimes}} Y^{\theta} .
$$

On the other hand, if $\omega \leq \gamma$, since $l_{1}\left([1, \gamma], X^{*}\right)$ has the approximation property (see for instance 2, page 285]), it follows from [3, Proposition 5.3] that

$$
\mathcal{K}\left(X^{\gamma}, Y^{\theta}\right) \sim l_{1}\left([1, \gamma], X^{*}\right) \hat{\hat{\otimes}} Y^{\theta} .
$$

Now suppose that $\beta<\alpha^{\omega}$. We have by Theorem 1.1 that $\mathbb{R}^{\alpha} \sim \mathbb{R}^{\beta}$ and thus $Y^{\alpha} \sim Y^{\beta}$. Hence according to (2) and (3) and our hypothesis on the cardinalities of $\xi$ and $\eta$, we conclude that $\mathcal{K}\left(X^{\xi}, Y^{\alpha}\right) \sim \mathcal{K}\left(X^{\eta}, Y^{\beta}\right)$.

Conversely, assume that $\omega \leq \alpha \leq \beta<\omega_{1}$ and $\mathcal{K}\left(X^{\xi}, Y^{\alpha}\right) \sim \mathcal{K}\left(X^{\eta}, Y^{\beta}\right)$. Then bearing in mind (2) and (3) we conclude that

$$
\mathbb{R}^{\beta} \hookrightarrow Y^{\beta} \hookrightarrow l_{1}\left([1, \eta], X^{*}\right) \hat{\hat{\otimes}} Y^{\beta} \hookrightarrow l_{1}\left([1, \xi], X^{*}\right) \hat{\hat{\otimes}} Y^{\alpha} \sim\left(l_{1}\left([1, \xi], X^{*}\right) \hat{\hat{\otimes}} Y\right)^{\alpha} .
$$

Next we distinguish two cases: $\alpha<\omega^{\omega}$ and $\omega^{\omega} \leq \alpha$.

Case 1. $\alpha<\omega^{\omega}$. We thus obtain from Theorem 1.1 that $\mathbb{R}^{\alpha} \sim \mathbb{R}^{\omega}$. So, by (4) we infer that

$$
\mathbb{R}^{\beta} \hookrightarrow\left(l_{1}\left([1, \xi], X^{*}\right) \hat{\hat{\otimes}} Y\right)^{\alpha} \sim\left(l_{1}\left([1, \xi], X^{*}\right) \hat{\hat{\otimes}} Y\right)^{\omega} \sim l_{1}\left([1, \xi], X^{*}\right) \hat{\hat{\otimes}} Y^{\omega} .
$$

Then, it follows from Proposition 2.2 that $\beta<\omega^{\omega}$. Again, an appeal to Theorem 1.1 tells us that $\mathbb{R}^{\beta} \sim \mathbb{R}^{\omega}$ and $\beta<\alpha^{\omega}$. 
Case 2. $\omega^{\omega} \leq \alpha$. In this case, by Proposition 2.1, $l_{1}\left([1, \xi], X^{*}\right) \hat{\hat{\otimes}} Y$ contains no subspace isomorphic to $\mathbb{R}^{\omega^{\omega}}$. Consequently, by (3) and Proposition $2.2, \mathbb{R}^{\beta} \hookrightarrow \mathbb{R}^{\alpha}$. Once again by Theorem 1.1, we get that $\beta<\alpha^{\omega}$. Thus the theorem is established.

\section{REFERENCES}

1. C. Bessaga, A. Pełczyński, Spaces of continuous functions. IV, Studia Math. XIX (1960), 53-61. MR.0113132 (22:3971)

2. P.G. Casazza, Approximation properties. Handbook of the geometry of Banach spaces. I, North-Holland Publishing Co., Amsterdam (2001), 271-316. MR.1863695 (2003f:46012)

3. A. Defant, K. Floret, Tensor norms and operator ideals, Math. Studies, 176, North-Holland, Amsterdam (1993). MR1209438 (94e:46130)

4. J. Diestel, J.J.J.R. Uhl, Vector Measures, Mathematical Surveys, 15, Amer. Math. Soc., Providence, RI (1977). MR0453964 (56:12216)

5. F. Lust, Produits tensoriels projectifs d'espaces de Banach faiblement séquentiellement complets, Colloq. Math. 36 (1976), 2, 255-267. MR0438153(55:11072)

6. E. M. Galego, Banach spaces of continuous vector-valued functions of ordinals, Proc. Edinb. Math. Soc. (2) 44 (2001), 1, 49-62. MR1879208 (2002k:46064)

7. E. M. Galego, On subspaces and quotients of Banach spaces $C(K, X)$, Monatsh. Math. 136 (2002), 2, 87-97. MR1914222 (2003g:46038)

8. E. M. Galego, On isomorphic classifications of space of compact operators, Proc. Amer. Math. Soc. 137 (2009), 10, 3335-3342. MR2515403 (2010d:46010)

9. E. M. Galego, Complete isomorphic classifications of some spaces of compact operators, Proc. Amer. Math. Soc. 138 (2010), 2, 725-736. MR2557189

10. P. Harmand, D. Werner, W. Werner, M-ideals in Banach spaces and Banach algebras, Lecture Notes in Math, 1547, Springer (1993). MR1238713 (94k:46022)

11. R. Haydon, E. Odell, H. Rosenthal, On certain classes of Baire-1 functions with applications to Banach space theory, Functional Analysis, Lecture Notes in Math., 1470, Springer (1991), 1-35. MR1126734 (92h:46018)

12. J. Lindenstrauss, L. Tzafriri, Classical Banach spaces. I. Sequence spaces, Springer-Verlag, Berlin-New York (1977). MR0500056 (58:17766)

13. J. Lindenstrauss, L. Tzafriri, Classical Banach spaces. II. Function spaces, Springer-Verlag, Berlin-New York (1979). MR540367 (81c:46001)

14. A. Pełczyński, A connection between weakly unconditional convergence and weak completeness of Banach spaces, Bull. Acad. Pol. Sci. 6 (1958), 251-253. MR0115072 (22:5875)

15. C. Samuel, On spaces of operators on $C(Q)$ spaces ( $Q$ countable metric space), Proc. Amer. Math. Soc. 137 (2009), 3, 965-970. MR2457436 (2009m:46004)

Department of Mathematics, University of São Paulo, São Paulo, Brazil 05508-090

E-mail address: eloi@ime.usp.br 